\title{
A Family of Einstein Randers Metrics
}

\author{
B. Najafi and A. Tayebi
}

November 2, 2018

\begin{abstract}
Using Hawking Taub-NUT metric $g_{a}$ on $\mathbf{R}^{4}$, where $a$ is a positive real number and finding a 4-parameter family of Killing vector fields of $\left(\mathbf{R}^{4}, g_{a}\right)$, we construct a 5-parameter family of Einstein Randers metrics with non-constant flag curvature.
\end{abstract}

Keywords: Hawking Taub-NUT metric, Flag curvature, Randers Einstein metric.

\section{Introduction}

The Taub-Newman-Unti-Tamburino (Taub-NUT) metrics were found by Taub 9] and extended by Newman-Unti-Tamburino [7. The Euclidean Taub-NUT metric has lately attracted much attention in physics. Hawking has suggested that the Euclidean Taub-NUT metric might give rise to the gravitational analog of the Yang-Mills instanton [6. This metric is the space part of the line element of the celebrated Kaluza-Klein monopole of Gross and Perry and Sorkin. The Taub-NUT family of metrics is also involved in many other modern studies in physics like strings, membranes, etc.

On the other hand, a rich and in some sense one of the best classes of Riemannian metrics is Einstein metrics, i.e, the solutions of the equation $R i c_{i j}-\lambda g_{i j}=0$ for some scalar $\lambda$. Einstein manifolds are not only interesting in physics but are also related to many important topics of Riemannian geometry such as self-dual manifolds of dimension four. In dimension three, being Riemannian Einstein metric is equivalent to being of constant sectional curvature. S. S. Chern conjectured that this equivalency is not true for Finslerian Einstein metrics. In [1], D. Bao and C. Robles classified Einstein Randers metrics in every dimension and showed that in dimension three, any Einstein Randers metric is of constant flag curvature (a natural generalization of sectional curvature) and vice versa. But for general Finslerian Einstein metrics, Chern's conjecture remains unsolved.

After classifying 3-dimensional Einstein metrics of Randers type, it is natural to classify 4-dimensional Einstein Randers metrics. The reasonable step is constructing as many as possible concrete examples. In this paper, we construct a 5-parameter family of 4-dimensional Randers Einstein metrics with 
non-constant flag curvature. This family contains the family of Randers Einstein metrics constructed in [5] as special case.

\section{Preliminaries}

Let $F$ be a Finsler metric on an $n$-dimensional manifold $M$, and $T M_{0}$ be its slant tangent space. In a local coordinate $\left(x^{i}, y^{i}\right)$, the vector filed $G=y^{i} \frac{\partial}{\partial x^{i}}-2 G^{i} \frac{\partial}{\partial y^{i}}$ is a global vector field on $T M_{0}$, where $G^{i}=G^{i}(x, y)$ are local functions on $T M_{0}$ satisfying $G^{i}(x, \lambda y)=\lambda^{2} G^{i}(x, y)$, for all $\lambda>0$. The vector field $G$ is called the associated spray to $(M, F)$.

The Riemann tensor can be viewed as a family of endomorphisms on tangent spaces: $\mathbf{R}_{y}(u):=\left.R_{k}^{i}(x, y) u^{k} \frac{\partial}{\partial x^{i}}\right|_{x}$, where $u=\left.u^{i} \frac{\partial}{\partial x^{i}}\right|_{x} \in T_{x} M$. The coefficients $R_{k}^{i}=R_{k}^{i}(x, y)$ are given by

$$
R_{k}^{i}=2 \frac{\partial G^{i}}{\partial x^{k}}-y^{j} \frac{\partial^{2} G^{i}}{\partial x^{j} \partial y^{k}}+2 G^{j} \frac{\partial^{2} G^{i}}{\partial y^{j} \partial y^{k}}-\frac{\partial G^{i}}{\partial y^{j}} \frac{\partial G^{j}}{\partial y^{k}} .
$$

For a tangent plane $P=\operatorname{span}(y, u) \subset T_{p} M$, the flag curvature of the flag $P$ with the flag pole $y$ is defined by

$$
K(P, y)=\frac{g_{y}\left(\mathbf{R}_{y}(u), u\right)}{g_{y}(y, y) g_{y}(u, u)-g_{y}(u, y) g_{y}(u, y)} .
$$

If $F$ is Riemannian, i.e., $g=g_{y}$ is independent of $y$, then the flag curvature $K(P, y)=K(P)$ is independent of $y \in P$ and it is the sectional curvature of $P$. Thus the flag curvature is an analogue of the sectional curvature in Riemannian geometry. $\mathrm{F}$ is said to be of constant flag curvature $\lambda$, if for any non-zero tangent vector $y \in T_{x} M$ and any flag $P$ containing $y, K(P, y)=\lambda$ is constant, or equivalently,

$$
\mathbf{R}_{y}=\lambda F^{2}(y)\left\{I-g_{y}(y, .) y\right\}, \quad y \in T_{x} M, \quad x \in M
$$

where $I: T_{x} M \rightarrow T_{x} M$ denotes the identity map and $g_{y}(y,)=.\frac{1}{2}\left[F^{2}\right]_{y^{i}} d x^{i}$.

The trace of $\mathbf{R}_{y}$ is denoted by $\operatorname{Ric}(y)$ and called the Ricci curvature. Hence, $\operatorname{Ric}(x, y)=R_{i}^{i}(x, y)$. A Finsler metric $F$ is said to be an Einstein metric if there is a scalar function $K=K(x)$ on $M$ such that Ric $=(n-1) K F^{2}$.

In [10, Wood constructs the following 4-dimensional Riemannian metric. Let $\left(N^{3}, h\right)$ be an oriented 3-dimensional Riemannian manifold with constant curvature. Let $M^{4}:=\mathbf{R} \times N^{3}$ and $\phi: M^{4} \rightarrow N^{3}$ be the projection on the second factor. Suppose that $u$ is a positive smooth function and $A$ a 1 -form on $N^{3}$, then the tensor $g$ on $M^{4}$ given by

$$
g:=u \phi^{*}(h)+u^{-1}(d t+A)^{2}
$$

is a Riemannian metric on $M^{4}$. In [4], Gibbons and Hawking prove that $\left(M^{4}, g\right)$ is an Einstein manifold if and only if the monopole equation $d u=* d A$ holds and $\left(N^{3}, h\right)$ is flat, in which case $g$ is Ricci-flat. 
Let us consider the following harmonic function on $\mathbf{R}^{3}-\{0\}$

$$
u_{a}(x):=\frac{1}{4}\left(\frac{1}{|x|}+a\right),
$$

where $a$ is a non-negative real number. Then the above construction gives the Hawking Taub-NUT metric $g_{a}(a>0)$ or the standard metric $g_{0}(a=0)$. A direct computation yields the following explicit formula for $g_{a}$

$$
g_{a}=\left(a|x|^{2}+1\right) g_{0}-\frac{a\left(a|x|^{2}+2\right)}{a|x|^{2}+1} \omega^{2},
$$

where $g_{0}$ is the standard metric and

$$
\omega=-x^{2} d x^{1}+x^{1} d x^{2}-x^{4} d x^{3}+x^{3} d x^{4}
$$

is a 1 -form on $\mathbf{R}^{4}$ (see [10] for more details). Hence the metric $g_{a}$ extends to the whole $\mathbf{R}^{4}$.

A Randers metric on a manifold $M$ is a Finsler metric in the following form

$$
F=\alpha+\beta,
$$

where $\alpha=\sqrt{a_{i j}(x) y^{i} y^{j}}$ is a Riemannian metric and $\beta=b_{i}(x) y^{i}$ a 1 -form on $M$. A nice description of Randers metrics is given by Zermelo navigation representation. Given a Riemannian metric $g=\sqrt{g_{i j} y^{i} y^{j}}$ and a vector field $V=V^{i} \frac{\partial}{\partial x^{i}}$, set $V_{i}:=g_{i j} V^{i}$ and $\|V\|_{g}:=\sqrt{V_{i} V^{i}}$. Then the pair $(g, V)$ with $\|V\|_{g}<1$ is called the Zermelo navigation representation (or simply navigation representation) of the Randers metric (44) if and only if the following relations hold

$$
a_{i j}=\frac{g_{i j}}{1-\|V\|_{g}^{2}}+\frac{V_{i} V_{j}}{\left(1-\|V\|_{g}^{2}\right)^{2}}, \quad b_{i}=-\frac{V_{i}}{1-\|V\|_{g}^{2}} .
$$

It is easy to see that, if $(g, V)$ is the navigation representation of $F=\alpha+\beta$, then the following hold

$$
F(x, y)=g(x, y-F(x, y) V(x)) .
$$

Many curvature properties of Randers metrics can be expressed in a geometrical way via their navigation representations. D. Bao and C. Robles use this method and characterize Einstein Randers metrics as follows [8].

Proposition 2.1 Suppose that a Randers metric $F=\alpha+\beta$ has the navigation representation $(g, V)$ on a manifold $M$. Then $F$ is an Einstein metric with $\operatorname{Ric}(x, y)=(n-1) K(x) F^{2}(x, y)$ if and only if

i) $V$ is a homothetic vector field of $g$ with $\mathcal{L}_{V}(g)=-4 c g$, where $\mathcal{L}_{V}$ denotes the Lie derivative along $V$.

ii) $g$ is Einstein metric with $\widetilde{R i c}=(n-1)\left\{K(x)+c^{2}\right\} g$, where $\widetilde{R i c}$ is the Ricci curvature of $g$. In particular, $K(x)=$ const if $n \geq 3$. 


\section{Construction of the Einstein Finsler Metrics}

Rewrite the Hawking Taub-NUT metric $g_{a}$ as

$$
g_{a}=g_{i j} d x^{i} d x^{j}
$$

where

$$
\left(g_{i j}\right)=\left(\begin{array}{cccc}
B-A\left(x^{2}\right)^{2} & A x^{1} x^{2} & -A x^{2} x^{4} & A x^{2} x^{3} \\
A x^{1} x^{2} & B-A\left(x^{1}\right)^{2} & A x^{1} x^{4} & -A x^{1} x^{3} \\
-A x^{2} x^{4} & A x^{1} x^{4} & B-A\left(x^{4}\right)^{2} & A x^{3} x^{4} \\
A x^{2} x^{3} & -A x^{1} x^{3} & A x^{3} x^{4} & B-A\left(x^{3}\right)^{2}
\end{array}\right)
$$

and $B=B(x)=a|x|^{2}+1, \quad A=A(x)=a\left(1+\frac{1}{B}\right)$.

The key observation is this: Suppose that $f: \mathbf{R}^{4} \rightarrow \mathbf{R}^{4}$ is an isometry of $g_{0}$, preserves the 1 -form $\omega$ given by (3) and $f(0)=0$. Then, it is easy to see that $f$ is also an isometry of $g_{a}$. Now we classify those Killing vector fields of $g_{0}$ which are also Killing vector fields with respect to $g_{a}$ and have the origin as a critical point. First, recall the following characterization theorem 3 .

Proposition 3.1 Let $X=X^{i} \frac{\partial}{\partial x^{i}}$ be a vector field on $\mathbf{R}^{4}$. Then $X$ is a Killing vector field of $g_{0}$ if and only if $X^{i}=Q_{j}^{i} x^{j}+C^{i}$, where $\left(Q_{j}^{i}\right)$ is an anti-symmetric matrix, $\left(C^{i}\right)$ is a constant vector.

Using Proposition 3.1, we find a 4-parameter family of Killing vector fields of $g_{a}$, and consequently a 5-parameter family of Einstein Randers metric with non-constant flag curvature. More precisely, with the notation of Proposition 3.1. we have the following.

Theorem 3.2 Let $X=X^{i} \frac{\partial}{\partial x^{i}}$ be a Killing vector field on $\left(\mathbf{R}^{4}, g_{0}\right)$. Then $X$ is a Killing vector field on $\left(\mathbf{R}^{4}, g_{a}\right)$ if and only if $\left(C^{i}\right)=(0)$ and the matrix $\left(Q_{j}^{i}\right)$ is in the following form

$$
\left(\begin{array}{cccc}
0 & m & s & r \\
-m & 0 & -r & s \\
-s & r & 0 & n \\
-r & -s & -n & 0
\end{array}\right)
$$

where $m, n, r$ and $s$ are constant real numbers.

Proof. Using the geometric meaning of Lie derivation of vector fields, the one parameter groups of the vector field $X=X^{i} \frac{\partial}{\partial x^{i}}$ on $\mathbf{R}^{4}$ preserves the 1 -form $\omega$ if and only if $\mathcal{L}_{X}(\omega)=0$. A direct computation implies that $\mathcal{L}_{X}(\omega)=0$ is equivalent to the following system of PDEs

$$
\begin{cases}-X^{2}-x^{2} \frac{\partial X^{1}}{\partial x^{1}}+x^{1} \frac{\partial X^{2}}{\partial x^{1}}-x^{4} \frac{\partial X^{3}}{\partial x^{1}}+x^{3} \frac{\partial X^{4}}{\partial x^{1}} & =0 \\ -x^{2} \frac{\partial X^{1}}{\partial x^{2}}+X^{1}+x^{1} \frac{\partial X^{2}}{\partial x^{2}}-x^{4} \frac{\partial X^{3}}{\partial x^{2}}+x^{3} \frac{\partial X^{4}}{\partial x^{2}} & =0 \\ -x^{2} \frac{\partial X^{1}}{\partial x^{3}}+x^{1} \frac{\partial X^{2}}{\partial x^{3}}-X^{4}-x^{4} \frac{\partial X^{3}}{\partial x^{3}}+x^{3} \frac{\partial X^{4}}{\partial x^{3}}=0 \\ -x^{2} \frac{\partial X^{1}}{\partial x^{4}}+x^{1} \frac{\partial X^{2}}{\partial x^{4}}-x^{4} \frac{\partial X^{3}}{\partial x^{4}}+X^{3}+x^{3} \frac{\partial X^{4}}{\partial x^{4}}=0\end{cases}
$$

By $\frac{\partial X^{i}}{\partial x^{i}}=\sigma$ and $\frac{\partial X^{i}}{\partial x^{j}}=Q_{j}^{i}$ for distinct $i$ and $j$, we get the result. Q.E.D. 
Using Proposition 2.1, we complete the construction in three cases.

\section{$3.1 \mathrm{r}=0, \mathrm{~s}=0$.}

If we put $Q_{2}^{1}=-m$ and $Q_{4}^{3}=-n$, where $m$ and $n$ are constant real numbers, then the vector field $X$ reduces to $W_{m, n}$ which is introduced by E. Guo, X. Mo, and X. Zhang in [5]. Using navigation representation $\left(g_{a}, W_{m, n}\right)$, a 3parameter family of Einstein Randers metric with non-constant flag curvature is constructed on the $\Omega=\left\{x \in \mathbf{R}^{4} \mid f(x)<1\right\}$, where

$$
f(x):=\left|W_{m, n}\right|_{g_{a}}=\frac{|x|^{2}}{1+a|x|^{2}}\left(p+2 a|m-n||x|^{2}+a^{2}|m-n||x|^{4}\right) .
$$

For more details see [5].

\section{$3.2 \mathrm{~m}=0, \mathrm{n}=0, \mathrm{~s}=0$.}

In this case, the vector field $X$ reduces to the vector field $V_{r}$ given by

$$
V_{r}:=r\left(x^{4} \partial_{1}-x^{3} \partial_{2}+x^{2} \partial_{3}-x^{1} \partial_{4}\right),
$$

where $r$ is a constant real number. Here, we construct Einstein Randers metric with non-constant flag curvature by using navigation representation $\left(g_{a}, V_{r}\right)$. First, we find the sufficient condition producing Einstein-Finsler metrics, i.e., $\left\|V_{r}\right\|_{g_{a}}<1$.

We have $V^{1}=r x^{4}, V^{2}=-r x^{3}, V^{3}=r x^{2}$, and $V^{4}=-r x^{1}$. A direct computation yields

$$
V_{i}:=g_{i j} V^{j}=\mu V^{i}
$$

where $\mu=\frac{1}{a|x|^{2}+1}$. Therefore, we get

$$
\left\|V_{r}\right\|_{g_{a}}^{2}=V_{i} V^{i}=\mu r^{2}|x|^{2} .
$$

If $r^{2}>a$, then define $\Omega_{a, r}=\left\{x \in \mathbf{R}^{4}|| x \mid<\frac{1}{\sqrt{r^{2}-a}}\right\}$. Otherwise, define $\Omega_{a, r}=\mathbf{R}^{4}$.

Theorem 3.3 Let $F=\alpha+\beta$ be a Randers metric be given by the navigation representation $\left(g_{a}, V_{r}\right)$. Then $F$ is an Einstein metric with non-constant flag curvature on $\Omega_{a, r}$.

Proof. From (9), we get $\left\|V_{r}\right\|_{g_{a}}<1$ on $\Omega_{a, r}$. Hence $F$ is actually a Randers metric on $\Omega_{a, r}$.

In [2, D. Bao and C. Robles prove that a Randers metric with navigation representation $(g, V)$ is of constant flag curvature if and only if $g$ is of constant sectional curvature and $V$ is a homothetic vector field with respect to $g$. The Taub-NUT metric is a Ricci flat metric on $\mathbf{R}^{4}$ which is not flat [6]. Hence 
$g_{a}$ is not of constant sectional curvature. Therefore $F$ is not of constant flag curvature.

It is sufficient to prove that $F$ is Einstein metric. Note that the Hawking Taub-NUT metric $g_{a}$ for all $a>0$ is an Einstein metric [10. From Theorem 3.2. we see that $V_{r}$ is a Killing vector field with respect to $g_{a}$. As an immediate consequence of Proposition 2.1, we conclude that $F$ is an Einstein metric. This completes the proof.

Q.E.D.

\section{$3.3 \mathrm{~m}=0, \mathrm{n}=0, \mathrm{r}=0$.}

In this case, the vector field $X$ reduces to the vector field $U_{s}$ given by

$$
U_{s}:=s\left(x^{3} \partial_{1}+x^{4} \partial_{2}-x^{1} \partial_{3}-x^{2} \partial_{4}\right),
$$

where $s$ is a constant real number. We have

$$
U^{1}=s x^{3}, U^{2}=s x^{4}, U^{3}=-s x^{1}, U^{4}=-s x^{2} .
$$

Using a Maple program, we get

$$
\left\|U_{s}\right\|_{g_{a}}^{2}=\mu s^{2}\left\{\left(1+8 a^{2} \prod_{i=1}^{4} x^{i}\right)|x|^{2}+2 a|x|_{4}^{4}+a^{2}|x|_{6}^{6}+16 a \prod_{i=1}^{4} x^{i}+f(x)\right\},
$$

where $|\cdot|_{k}$ denotes the $k$-norm on $\mathbf{R}^{4}$ and the scalar function $f$ is given by

$$
\begin{aligned}
f(x)= & -a^{2}\left(x_{1}^{2} x_{4}^{4}+x_{2}^{4} x_{3}^{2}+x_{2}^{2} x_{3}^{4}+x_{1}^{4} x_{4}^{2}\right) \\
& +3 a^{2}\left(x_{1}^{2} x_{2}^{4}+x_{4}^{2} x_{2}^{4}+x_{1}^{2} x_{3}^{4}+x_{3}^{2} x_{4}^{4}+x_{3}^{4} x_{4}^{2}+x_{1}^{4} x_{2}^{2}+x_{2}^{2} x_{4}^{4}+x_{3}^{2} x_{1}^{4}\right) \\
& +4 a\left(x_{1}^{2} x_{3}^{2}-x_{2}^{2} x_{3}^{2}+x_{2}^{2} x_{1}^{2}+x_{4}^{2} x_{3}^{2}-x_{4}^{2} x_{1}^{2}+x_{4}^{2} x_{2}^{2}\right) \\
& +2 a^{2}\left(x_{1}^{2} x_{3}^{2} x_{4}^{2}+x_{2}^{2} x_{3}^{2} x_{4}^{2}+x_{1}^{2} x_{2}^{2} x_{3}^{2}+x_{1}^{2} x_{2}^{2} x_{4}^{2}\right),
\end{aligned}
$$

in which $x_{i}^{k}$ means $\left(x^{i}\right)^{k}$.

Set

$$
\widetilde{\Omega}_{a, s}:=\left\{x \in \mathbf{R}^{4} \mid\left\|U_{s}\right\|_{g_{a}}<1\right\} .
$$

Then we have the following theorem.

Theorem 3.4 Let $F=\alpha+\beta$ be a Randers metric given by the navigation representation $\left(g_{a}, U_{s}\right)$. Then $F$ is an Einstein metric with non-constant flag curvature on $\widetilde{\Omega}_{a, s}$.

These concrete examples of four dimensional Randers Einstein metrics show that this class of Randers metrics is large and of course far from being completely classified. 


\section{References}

[1] D. Bao, C. Robles: On Randers spaces of constant flag curvature, Rep. Math. Phys. 51 (2003), 9-42.

[2] D. Bao, C. Robles: Ricci and flag curvature in Finsler geometry, in A sampler of Riemann-Finsler Geometry, Math. Sci. Res. Ins. Publ., Vol. 50, Cambridge Univ. Press, Cambridge, 2004, 197-259.

[3] D. Bao, C. Robles, and Z. Shen: Zermelo navigation on Riemannain manifolds, J. Diff. Geom, 66 (2004), 377-435.

[4] G. W. Gibbons and S. W. Hawking: Gravitational multi-instantons. Phys. Lett. B 78 (1978), 430-432.

[5] E. Guo, X. Mo and X. Zhang: The explicit construction of Einstein Finsler metrics with non-constant flag curvature, Symmetry, Integrabilty and Geometry: Methods and Applications, 5 (2009), 045.

[6] S. W. Hawking: Gravitational instantons. Phys. Lett. A 60 (1977), no. $2,81-83$.

[7] E. Newman, L. Tamburino and T. Unti, Empty-space generalization of the Schwartzschild metric, J. Math. Phys. 4 (1963) 915-923.

[8] C. Robles: Einstein metrics of Randers type, Ph.D Thesis, British Columbia University, Canada, 2003.

[9] A. H. Taub: Empty space-times admitting a three parameter group of motions, Ann. of Math. 53 (1951) 472-490.

[10] J. C. Wood: Harmonic morphism between Riemannian manifolds, in Modern Trends in Geometry and Topology, Cluj Univ. Press, ClujNapoca, 2006,397-414.

Behzad Najafi

Faculty of Science, Department of Mathematics

Shahed University of Tehran

Tehran. Iran

Email: najafi@shahed.ac.ir

Akbar Tayebi

Faculty of Science, Department of Mathematics

Qom University

Qom. Iran

Email: akbar.tayebi@gmail.com 